\title{
Clinical Progress Note: Pediatric Acute Kidney Injury
}

\author{
Jean-Philippe Roy, MD*; Catherine S Forster, MD, MS²
}

${ }^{1}$ Center for Acute Care Nephrology, Cincinnati Children's Hospital Medical Center, Cincinnati, Ohio; ${ }^{2}$ Division of Hospitalist Medicine, Children's National Medical Center, Washington, DC.

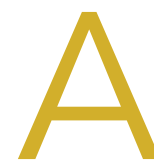

cute kidney injury (AKI) occurs in 5\%-30\% of noncritically ill hospitalized children. ${ }^{1}$ Initially thought to be simply a symptom of more severe pathologies, it is now recognized that $\mathrm{AKI}$ independently increases mortality and is associated with the development of chronic kidney disease (CKD), even in children. ${ }^{2}$ The wide acceptance of the Kidney Disease Improving Global Outcome (KDIGO) diagnostic criteria has enabled a more uniform definition of AKI from both clinical and research perspectives. ${ }^{2} \mathrm{~A}$ better understanding of the pathophysiology and risk factors for AKI has led to new methods for early detection and prevention efforts. While serum creatinine $(\mathrm{SCr})$ was historically one of the sole markers of AKI, novel biomarkers can facilitate earlier diagnosis of AKI, identify subclinical AKI, and guide clinical management. This clinical practice update addresses the latest clinical advances in risk assessment, diagnosis, and prevention of pediatric $A K I$, with a focus on AKI biomarkers.

\section{DIAGNOSIS, BIOMARKERS, AND DEFINITION}

Several sets of criteria have been used to diagnose AKI. The KDIGO classification, based on a systematic review of the literature and developed through expert consensus, is the current recommended definition. ${ }^{3}$ Increasing AKI stage, as defined by the KDIGO classification, is associated with increased mortality, the need for renal replacement therapy, length of stay, and $C K D$, thus underscoring the importance of accurate classification. ${ }^{3}$ Stage $1 \mathrm{AKI}$ is defined by a rise in $\mathrm{SCr}$ of $\geq 0.3 \mathrm{mg} / \mathrm{dL}, 1.5$ 1.9 times the baseline $\mathrm{SCr}$, or urine output $<0.5 \mathrm{ml} / \mathrm{kg} / \mathrm{h}$ for six to 12 hours; stage 2 by a rise of $\geq 2.0-2.9$ times the baseline $\mathrm{SCr}$ or urine output $<0.5 \mathrm{ml} / \mathrm{kg} / \mathrm{h}$ for $>12$ hours; and stage 3 by a rise of $\geq 4.0 \mathrm{mg} / \mathrm{dL}$, $\geq$ three times the baseline $\mathrm{SCr}$, initiation of renal replacement therapy, urine output $<0.3 \mathrm{ml} / \mathrm{kg} / \mathrm{h}$ for $\geq 24$ hours, or anuria $\geq 12$ hours. However, these criteria rely on $\mathrm{SCr}$, which is a suboptimal marker of renal dysfunction, as it rises only once the glomerular filtration rate (GFR) has already decreased, in some cases by as much as $50 \%$. Additionally, interpretation of $\mathrm{SCr}$ in the diagnosis of $\mathrm{AKI}$ requires a prior $\mathrm{Scr}$ measurement to determine the magnitude of change from the baseline value, which is often lacking in children. To mitigate this limitation, different formulas exist to estimate a baseline $\mathrm{SCr}$ value based on height or age, an approach that

*Corresponding Author: Jean-Philippe Roy, MD; E-mail: jean-philippe.roy@ cchmc.org; Telephone: 514-622-8331.

Published online first August 21, 2019.

Received: April 3, 2019; Revised: June 25, 2019; Accepted: Jun 28, 2019

(c) 2019 Society of Hospital Medicine DOI 10.12788/jhm.3280 assumes patients have preexisting normal renal function.

The limitations of $\mathrm{SCr}$ have led to interest in identifying more accurate biomarkers of AKI. Although many candidates have been identified, we will limit our discussion to those currently available for clinical use: serum cystatin $C$, urine neutrophil gelatinase-associated lipocalin (NGAL), urine TIMP-2, and urine IGFBP7 (Table). ${ }^{4.8}$ While urine NGAL and cystatin $C$ are measured individually, TIMP-2 and IGFBP7 are measured on the same panel and the product of their multiplied values is used for clinical guidance. While each of these biomarkers have good predictive accuracy for AKI when used independently, their combined use increases the accuracy of AKI diagnosis. These biomarkers can be divided into broad categories based on their utility as either functional markers or markers of injury. ${ }^{6}$ Serum cystatin C is a functional marker and as such can be used to estimate GFR more accurately than $\mathrm{SCr} .{ }^{9}$ Comparatively, urine NGAL is a marker of renal injury, while TIMP2 and IGFBP7 are markers of renal stress. These markers are not useful in estimating GFR, but rather aid in the prediction and diagnosis of AKI (Figure). Despite the limitations of $\mathrm{SCr}$, these biomarkers have yet to be incorporated into the diagnostic criteria. They have, however, helped to refine our understanding of the pathophysiology of AKI.

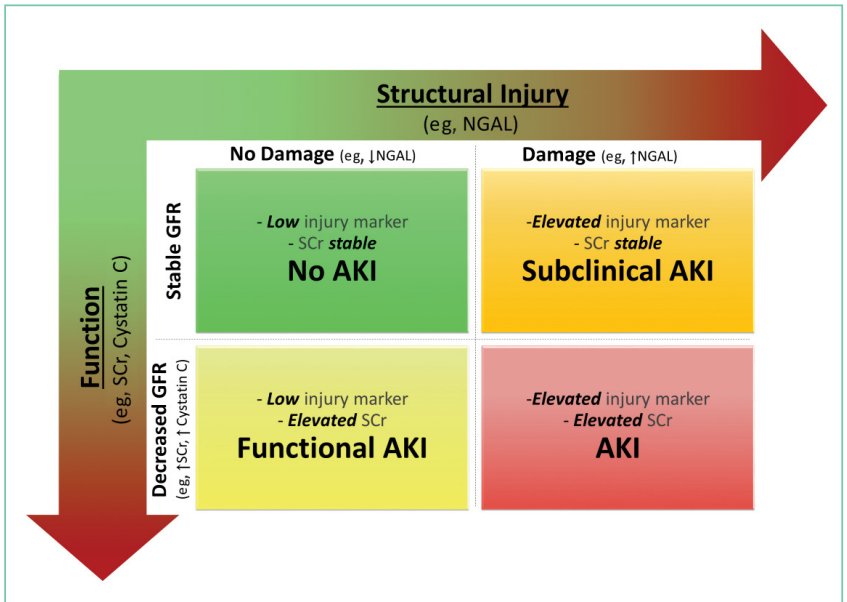

FIG. Biomarkers of structural injury (eg, urine NGAL) can be used in conjunction with biomarkers of function (eg, $\mathrm{SCr}$, cystatin $\mathrm{C}$ ) to increase the precision of the diagnosis of $\mathrm{AKI}$. While an elevation of $\mathrm{SCr}$ or cystatin $\mathrm{C}$ translates to a decrease in GFR, it can be due to either a functional hemodynamic process or an AKI (injury biomarkers low or elevated, respectively). Moreover, elevated injury biomarkers (eg, high urine NGAL), which indicate the presence of tissue injury, may be present without a corresponding rise in $\mathrm{SCr}$ if a significant decrease in GFR has not yet occurred. The latter scenario reflects a subclinical AKI. Adapted from Endre et al. ${ }^{6}$

Abbreviations: AKI, acute kidney injury; GFR, glomerular filtration rate; NGAL, neutrophil gelatinase-associated lipocalin; $\mathrm{SCr}$, serum creatinine. 
TABLE. Frequently Used Biomarkers for AKI Management

\begin{tabular}{|c|c|c|c|}
\hline & NGAL & $\begin{array}{l}\text { TIMP-2*IGFBP7 } \\
\left(\text { NephroCheck }^{\circledast}\right)\end{array}$ & Cystatin C \\
\hline Preferred Sample Type & Urine & Urine & Serum \\
\hline Pathway & $\begin{array}{l}\text { Siderophore secreted by tubular epithelial cells early } \\
\text { in AKI }\end{array}$ & G1 cell-cycle arrest proteins secreted by tubular cells & $\begin{array}{l}\text { Protein produced by all nucleated cells in the body that } \\
\text { is filtered at the level of the glomerulus and mostly } \\
\text { metabolized in the proximal tubule after filtration }\end{array}$ \\
\hline Clinical Implication & Structural damage & Cellular stress & Glomerular filtration rate \\
\hline General Clinical Use & $\begin{array}{l}\text { Distinguishes functional AKI from intrinsic AKI. Predicts } \\
\text { severe AKI in high-risk patients and worse outcomes in } \\
\text { critically ill patients }\end{array}$ & $\begin{array}{l}\text { Predicts AKI after injury or exposure and worse } \\
\text { outcome in critically ill patients }\end{array}$ & $\begin{array}{l}\text { Detects glomerular dysfunction and provides a more } \\
\text { accurate assessment of eGFR in certain conditions (eg, } \\
\text { low muscle mass, children, CKD) }\end{array}$ \\
\hline Advantages & $\begin{array}{l}\text { Can be used to track the clinical evolution of AKI. } \\
\text { Available as point of care }\end{array}$ & $\begin{array}{l}\text { Can predict the risk of AKI before elevation of injury } \\
\text { markers. Available as point of care }\end{array}$ & $\begin{array}{l}\text { Can be used alone or in combination with } \mathrm{SCr} \text { to } \\
\text { estimate GFR. Levels are largely unaffected by age, } \\
\text { gender, diet, or tubular secretion }\end{array}$ \\
\hline Limitations & $\begin{array}{l}\text { Sepsis and urinary tract infections can increase urinary } \\
\text { NGAL }\end{array}$ & $\begin{array}{l}\text { Peaks quickly (around } 4 \text { hours) after injury with a rapid } \\
\text { decline in levels }\end{array}$ & $\begin{array}{l}\text { Steroid use or hyperthyroidism can lead to } \\
\text { underestimation of GFR. Hypothyroidism can lead to } \\
\text { overestimation of GFR }\end{array}$ \\
\hline Clinical Scenario Example & $\begin{array}{l}\text { Differentiate functional from intrinsic AKI in a patient } \\
\text { admitted with diarrheal illness and concomitant } \\
\text { nephrotoxic exposure }\end{array}$ & $\begin{array}{l}\text { Identify postoperative cardiac patients at risk of } \\
\text { severe AKI for an early strict application of the KDIGO } \\
\text { guidelines with nephrotoxic exposure reduction }\end{array}$ & $\begin{array}{l}\text { Accurate medication adjustment in a pediatric patient } \\
\text { with medical complexity. Identify a decrease in eGFR in } \\
\text { a patient receiving chemotherapy with recent addition of } \\
\text { nephrotoxic antibiotics. }\end{array}$ \\
\hline
\end{tabular}

Table content summarized from multiple publications. ${ }^{48}$

Abbreviations: AKI, acute kidney injury; CKD, chronic kidney disease; ; eGFR, estimated glomerular filtration rate; NGAL, neutrophil gelatinase-associated lipocalin; KDIGO, Kidney Disease-Improving Global Outcomes; SCr, serum creatinine; TIMP-2*IGFBP7, tissue inhibitor of metalloproteinases 2 and insulin-like growth factor-binding protein 7.

AKI has classically been divided into three categories based on the etiology of injury, namely prerenal azotemia, intrinsic renal disease, and postrenal causes. The discovery of new biomarkers adds nuance to the classification of AKI. Two groups of biomarkers are particularly helpful in this regard: markers of structural injury (eg, NGAL) and functional markers (eg, cystatin C). The combination of these biomarkers with $\mathrm{SCr}$ has refined the categories of AKI (Figure). For example, NGAL can accurately distinguish between a rise in $\mathrm{SCr}$ due to functional $\mathrm{AKI}$, previously referred to as prerenal azotemia, and a rise in $\mathrm{SCr}$ due to intrinsic kidney injury. An elevation of structural injury biomarkers in the absence of a significant rise in $\mathrm{SCr}$ is referred to as subclinical AKI. Patients with subclinical AKI have worse outcomes than those without AKI but better outcomes than patients with AKI with elevation of both $\mathrm{SCr}$ and NGAL (Figure). 2,6 Time to resolution of AKI further refines our ability to predict prognosis and outcomes. Transient AKI, defined as resolution within 48 hours, is associated with a better prognosis than persistent AKI. Renal dysfunction lasting more than seven days but less than 90 days is referred to as acute kidney disease (AKD). While both transient $A K I$ and $A K D$ represent different entities on the continuum between AKI and CKD, further research is needed to better elucidate these classifications. ${ }^{2}$

\section{RISK STRATIFICATION}

The renal angina index (RAl) identifies critically ill children at high risk for AKI. The RAI combines traditional markers of AKI, such as a change in estimated creatinine clearance and fluid overload, with patient factors, including need for ventilation, inotropic support, and history of transplantation (solid organ or bone marrow) to identify those patients who are at high risk for severe AKI. Patients identified as high risk by the patient factors component of the RAl have a much lower threshold for both a decrease in creatinine clearance and fluid overload to be considered at risk for severe AKI, as these early signs are more likely to reflect an early impending severe AKI in this high-risk group. Conversely, patients that do not meet these patient factors are more likely to simply have a transient or functional AKI, and therefore have a higher threshold for both a change in creatinine clearance and fluid overload in order to be considered at high risk for severe AKI.

The RAI has been validated in the critical care setting as a method to predict severe AKI at day three of admission to the pediatric intensive care unit, with a negative predictive value of 92\%-99\% when the score is negative in the first 12 hours. ${ }^{10}$ In selected high-risk patients (RAl $\geq 8$ ), biomarkers become even more reliable for AKI prediction (eg, injury markers have an excellent area under the receiver operating characteristic curve (AUC) of 0.97 for severe AKI prediction in this high-risk group). ${ }^{11}$ While only validated for critically ill patients, the concept of renal angina is still applicable in the complex populations managed by hospitalists who practice outside of the intensive care unit setting. Early signs of renal dysfunction (eg, rising $\mathrm{SCr}$, fluid overload $\geq 5 \%$ ) in patients with risk factors (see below) should prompt a thorough evaluation, including urinalysis, daily $\mathrm{SCr}$, nephrotoxin avoidance, and tissue injury biomarkers, if available.

The risk factors for $\mathrm{AKI}$ are numerous and tend to potentiate one another. The most frequent predisposing comorbidities include CKD, heart failure or congenital heart diseases, transplantation (bone marrow or solid organs), and diabetes. Dis- 
ease-related factors include sepsis, cardiac surgery, cardio-pulmonary bypass, mechanical ventilation, and vasopressor use. Potentially modifiable factors include hypovolemia and multiple nephrotoxic exposures. ${ }^{2,3}$

Nephrotoxic medications are now among the most common causes of AKI in hospitalized children. ${ }^{12}$ Approximately $80 \%$ of children are exposed to at least one nephrotoxin during an inpatient admission. ${ }^{12}$ Exposure to a single nephrotoxic medication is sufficient to place a child at risk of $A K I$, and each additional nephrotoxin further increases the risk. ${ }^{12}$ While some drugs are routinely recognized to be nephrotoxic (eg, ibuprofen), others are commonly overlooked, notably certain antibiotics (eg, cefotaxime, ceftazidime, cefuroxime, nafcillin, and piperacillin) and anticonvulsants (eg, zonisamide). ${ }^{12}$ Furthermore, the combination of multiple nephrotoxins can potentiate the risk of AKI. For example, the combination of vancomycin and piperacillin/tazobactam increases the risk of $\mathrm{AKI}$ by 3.4 times compared with the combination of vancomycin with another antipseudomonal beta-lactam antibiotic. ${ }^{13}$

Adequate monitoring, including daily $\mathrm{SCr}$ measurements and risk awareness, are critical as nephrotoxin-associated AKI can be easily missed in the absence of routine $\mathrm{SCr}$ monitoring, especially since these children are typically nonoliguric ${ }^{12}$. Quality improvement efforts focused on obtaining daily $\mathrm{SCr}$ in patients exposed to either three or more nephrotoxins or three days of either aminoglycoside or vancomycin, even without concomitant exposure to other nephrotoxins, have shown success in decreasing both the number of nephrotoxins and the rate of nephrotoxin-associated $\mathrm{AKI}{ }^{12}$

While a significant injury cannot always be avoided, a mindful clinical approach and management can help to prevent some complications of AKI. An awareness of fluid status is critical, as fluid overload greater than $10 \%$ of the patient's weight independently increases the risk of mortality in both adults and children. ${ }^{14}$ To assess the risk of AKI progression and potential failure of conservative management with diuretics, a furosemide stress test (FST) is an easy, safe, and accessible functional assessment of tubular reserve in a patient without intravascular depletion. ${ }^{15}$ A growing body of literature in adults shows that FST-responders are less likely to progress to stage $3 \mathrm{AKI}$ or need renal replacement therapy than nonresponders. ${ }^{15}$ The FST is currently being investigated and standardized in children.

\section{CONCLUSION}

Research in AKI has made significant strides over the last few years. Nevertheless, many areas of research remain to be explored (eg, the impact of IV fluid type in the pediatric population, AKD characterization and impact on CKD development). $\mathrm{AKI}$ is common, associated with significant morbidity and mortality and, in some instances, preventable. While no targeted therapeutic options are currently under investigation, recent advances allow for better identification of high-risk patients and offer opportunities for impactful preventive approaches. Thoughtful use of nephrotoxic medications, early identification of patients at high risk for AKI, and accurate diagnosis and appropriate management of AKI are the recommended best practice.

Disclosures: The authors have nothing to disclose.

\section{References}

1. McGregor TL, Jones DP, Wang $L$, et al. Acute kidney injury incidence in noncritically ill hospitalized children, adolescents, and young adults: a retrospective observational study. Am J Kidney Dis. 2016;67(3):384-390. https://doi. org/10.1053/j.ajkd.2015.07.019.

2. Chawla LS, Bellomo R, Bihorac A, et al. Acute kidney disease and renal recovery: consensus report of the Acute Disease Quality Initiative (ADQI) 16 Workgroup. Nat Rev Nephrol. 2017;13(4):241-257. https://doi.org/10.1038/nrneph.2017.2.

3. Khwaja A. KDIGO clinical practice guidelines for acute kidney injury. Nephron Clin Pract. 2012;120(4):179-184. https://doi.org/10.1159/000339789.

4. Filho LT, Grande AJ, Colonetti T, Della ÉSP, da Rosa MI. Accuracy of neutrophil gelatinase-associated lipocalin for acute kidney injury diagnosis in children: systematic review and meta-analysis. Pediatr Nephrol. 2017;32(10):19791988. https://doi.org/10.1007/s00467-017-3704-6.

5. Levey AS, Inker LA. Assessment of glomerular filtration rate in health and disease: a state of the art review. Clin Pharmacol Ther. 2017;102(3):405-419. https://doi.org/10.1002/cpt.729.

6. Endre ZH, Kellum JA, Di Somma S, et al. Differential diagnosis of AKI in clinical practice by functional and damage biomarkers: workgroup statements from the tenth Acute Dialysis Quality Initiative Consensus Conference. Contrib Nephrol. 2013;182:30-44. https://doi.org/10.1159/000349964.

7. Su LJ, Li YM, Kellum JA, Peng ZY. Predictive value of cell cycle arrest biomarkers for cardiac surgery-associated acute kidney injury: a meta-analysis. Br J Anaesth. 2018;121(2):350-357. https://doi.org/10.1016/j.bja.2018.02.069.

8. Westhoff $\mathrm{JH}$, Tönshoff $\mathrm{B}$, Waldherr $\mathrm{S}$, et al. Urinary tissue inhibitor of metalloproteinase-2 (TIMP-2) · insulin-like growth factor-binding protein 7 (IGFBP7) predicts adverse outcome in pediatric acute kidney injury. PLoS One. 2015;10(11):1-16. https://doi.org/10.1371/journal.pone.0143628.

9. Berg UB, Nyman U, Bäck R, et al. New standardized cystatin C and creatinine GFR equations in children validated with inulin clearance. Pediatr Nephrol. 2015;30(8):1317-1326. https://doi.org/10.1007/s00467-015-3060-3.

10. Chawla LS, Goldstein SL, Kellum JA, Ronco C. Renal angina: concept and development of pretest probability assessment in acute kidney injury. Crit Care. 2015;19(1):93. https://doi.org/10.1186/s13054-015-0779-y.

11. Menon S, Goldstein SL, Mottes T, et al. Urinary biomarker incorporation into the renal angina index early in intensive care unit admission optimizes acute kidney injury prediction in critically ill children: a prospective cohort study. Nephrol Dial Transplant. 2016;31(4):586-594. https://doi.org/10.1093/ndt/gfv457.

12. Goldstein SL, Mottes T, Simpson K, et al. A sustained quality improvement program reduces nephrotoxic medication-associated acute kidney injury. Kidney Int. 2016;90(1):212-221. https://doi.org/10.1016/j.kint.2016.03.031.

13. Downes KJ, Cowden C, Laskin BL, et al. Association of acute kidney injury with concomitant vancomycin and piperacillin/tazobactam treatment among hospitalized children. JAMA Pediatr. 2017;19146:e173219-e173219. https:// doi.org/10.1001/JAMAPEDIATRICS.2017.3219

14. Naipaul A, Jefferson LS, Goldstein SL, Loftis LL, Zappitelli M, Arikan AA. Fluid overload is associated with impaired oxygenation and morbidity in critically ill children*. Pediatr Crit Care Med. 2011;13(3):253-258. https://doi. org/10.1097/pcc.0b013e31822882a3.

15. Lumlertgul N, Peerapornratana S, Trakarnvanich T, et al. Early versus standard initiation of renal replacement therapy in furosemide stress test non-responsive acute kidney injury patients (the FST trial). Crit Care. 2018;22(1):1-9. https://doi.org/10.1186/s13054-018-2021-1. 